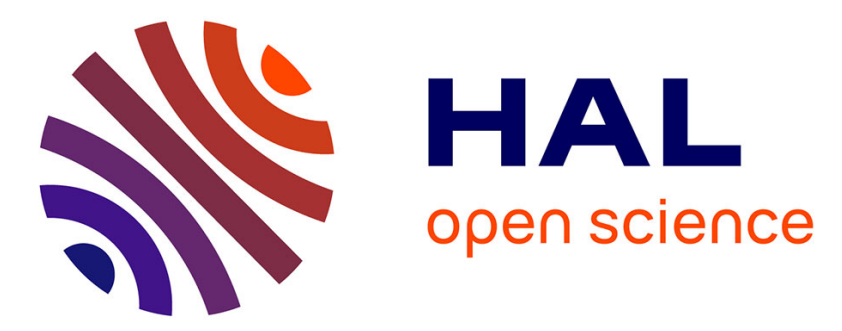

\title{
A simplified lumped model for the optimization of post-buckled beam architecture wideband generator
}

\author{
Weiqun Liu, Fabien Formosa, Adrien Badel, Guangdi Hu
}

\section{To cite this version:}

Weiqun Liu, Fabien Formosa, Adrien Badel, Guangdi Hu. A simplified lumped model for the optimization of post-buckled beam architecture wideband generator. Journal of Sound and Vibration, 2017, 409, pp.165-179. 10.1016/j.jsv.2017.07.049 . hal-01901522

\section{HAL Id: hal-01901522 \\ https://hal.science/hal-01901522}

Submitted on 23 Oct 2018

HAL is a multi-disciplinary open access archive for the deposit and dissemination of scientific research documents, whether they are published or not. The documents may come from teaching and research institutions in France or abroad, or from public or private research centers.
L'archive ouverte pluridisciplinaire HAL, est destinée au dépôt et à la diffusion de documents scientifiques de niveau recherche, publiés ou non, émanant des établissements d'enseignement et de recherche français ou étrangers, des laboratoires publics ou privés. 


\title{
A simplified lumped model for the optimization of post- buckled beam architecture wideband generator
}

\author{
Weiqun Liu ${ }^{1}$, Fabien Formosa ${ }^{2}$, Adrien Badel $^{2}$, Guangdi Hu${ }^{1}$ \\ ${ }^{1}$ School of Mechanical Engineering, Southwest Jiaotong University, 610031, Chengdu, China \\ ${ }^{2}$ Laboratoire SYMME, Université Savoie Mont Blanc, 74000, Annecy, France \\ Email : weiqunliu@home.swjtu.edu.cn
}

\begin{abstract}
Buckled beams structures are a classical kind of bistable energy harvesters which attract more and more interests because of their capability to scavenge energy over a large frequency band in comparison with linear generator. The usual modeling approach uses the Galerkin mode discretization method with relatively high complexity, while the simplification with a single-mode solution lacks accuracy. It stems on the optimization of the energy potential features to finally define the physical and geometrical parameters. Therefore, in this paper, a simple lumped model is proposed with explicit relationship between the potential shape and parameters to allow efficient design of bistable beams based generator. The accuracy of the approximation model is studied with the effectiveness of application analyzed. Moreover, an important fact, that the bending stiffness has little influence on the potential shape with low buckling level and the sectional area determined, is found. This feature extends the applicable range of the model by utilizing the design of high moment of inertia. Numerical investigations demonstrate that the proposed model is a simple and reliable tool for design. An optimization example of using the proposed model is demonstrated with satisfactory performance.
\end{abstract}

Keywords: Post-buckled beam; Bistable wideband generator; Simplified lumped model; Energy harvesting; Optimization and design

\section{Introduction}

\subsection{Background}

As an emerging technology, vibration energy harvesting has been an active research area in the past years because of its prospective applications [1]. It offers new possibilities for the development of autonomous sensor networks which are becoming more and more important in many industry fields, structure health monitoring, environment monitoring and protection, smart transportation etc. 
In order to make the harvester adapt to the variable vibration excitations of various application environments, the development of an energy harvester of wideband performance is attracting more and more interests. Numerous studies have been done in recent years [2-5]. Different mechanisms have been proposed: frequency-up conversion methods [6,7], resonance-tuning solutions [8], nonlinear oscillators [9-15], array or multi-mode harvesters [16-18], nonlinear energy sink [19, 20] etc.

Within the nonlinear generator group, bi-stable oscillator has been extensively studied $[21,22]$ due to its two favourable aspects: i) the frequency-up conversion effects associated to the snap-through motion in the low-frequency; ii) the bandwidth increase due to the nonlinear backbone responses. The first bistable generator was almost proposed at the same time by Cottone et al. using a piezoelectric inverted pendulum using magnetic repelling force [23] and Erturk et al. with a piezomagnetoelastic beam utilizing the attractive force of two magnets [24]. Numerous studies were further performed on similar structures with different arrangements or excitations [25-29]. Subsequently, Mann et al. presented a circular bistable electromagnetic harvester driven by non-contact magnetic repulsion [30] while Ferrari et al. showed a design of the bi-stable cantilever beam with a single magnet by using the attractive force from two separate poles [31]. Apart from the aforementioned structures that adopt the magnetic interaction mechanisms, the buckling effect is another common way of creating bi-stable generators. Arrieta et al. [32] and Betts et al. investigated a piezoelectric bi-stable composite plate and its optimal configuration used for vibration energy harvesting [33], while the present authors proposed a bi-stable BSM (BuckledSpring-Mass) generator architecture [34, 35]. One of the most well-known bi-stable structures of this type is the post-buckled beam. Masana and Daqaq [36] investigated the performance of the clamped-clamped beam in mono-stable and bi-stable cases for sweep excitations. An axially compressed beam shows the same characteristics as the hardening oscillator in the mono-stable case and the bi-stable Duffing oscillator ones after buckling. Cottone et al. discussed the post-buckled beam structure for piezoelectric [37] and electromagnetic [38] energy harvesting respectively. Sneller et al. [39] investigated a postbuckled piezoelectric beam with an attached centre mass to scavenge energy from the chirp excitations in consideration of increasing the probabilities of inter-well motions. More recently, Cottone et al. studied the effect of different boundary conditions on the piezoelectric post-buckled beam that the fixed-fixed case presents better performance than hinged cases [40]. In comparison with other bi-stable structures, the post-buckled beam is compact and easy to fabricate, which is especially favourable to achieve high power density.

The shape of the mechanical energy potential function (EPF) of the post-buckled beam plays an important role on the harvested power [36]. The structure geometrical and material parameter optimization is then to be derived from the chosen EPF for obtaining desired performance with a proper model required. The Galerkin modal discretization is a mostly used approach for the analysis of the postbuckled beams [36-40]. A single-mode model is often used in the literatures [37-39] for simplification 
instead of the more complex multi-mode solution that is difficult to analyse. However, as shown in [41], the single-mode model does not represent the real system well since the potential symmetry is lost in many cases. Furthermore, the explicit relationship between the shape of the post-buckled beam's EPF and the beam parameters has not been well established yet even with the single-mode model. As a consequence, the lack of a simple and accurate model hinders the optimization and design of the postbuckled beam generator.

\subsection{Geometry description of a potential representative architecture}

As a first example, the bistable oscillator and the transducer are supposed to be weakly coupled. Fig. 1 shows the general scheme of a potential realistic fixed-fixed post-buckled beam generator including an electromagnetic energy conversion. The latter will be typically considered as a damper for the mechanical non-linear oscillator. An inertial mass $M$ is placed at the centre of the beam to improve the dynamic responses. The geometry parameters are summarized in table 1 . As the horizontal axial force exceeds the critical buckling force, the beam becomes unstable at the original position and bends into one of the buckling symmetrical configuration as indicated by the solid and dashed shapes.

Table 1 Parameters for the post-buckled beam

\begin{tabular}{cccc}
\hline Parameter & Symbol & Parameter & Symbol \\
\hline Young's modulus $\left(\mathrm{N} / \mathrm{m}^{2}\right)$ & $E$ & Inertial mass $(\mathrm{kg})$ & $M$ \\
Density $\left(\mathrm{kg} / \mathrm{m}^{3}\right)$ & $\rho$ & Beam mass per unit of length $(\mathrm{kg} / \mathrm{m})$ & $m$ \\
Buckling level $(\mathrm{m})$ & $h_{p}$ & Cross section $\left(\mathrm{m}^{2}\right)$ & $A$ \\
Length of beam $(\mathrm{m})$ & $L$ & Quadratic momentum $\left(\mathrm{m}^{4}\right)$ & $I$ \\
Width $(\mathrm{m})$ & $W_{t}$ & Thickness $(\mathrm{m})$ & $h_{0}$ \\
\hline
\end{tabular}

In this paper, a simple lumped model for a post-buckled with a dominant inertial mass is derived from the Galerkin multi-mode solution by assuming the symmetric snap-through buckling behaviour. Its effectiveness is discussed in detail by analysing the influence of the antisymmetric buckling and comparing the solved beam shape and the potential function of the two models with the possible applicable situations given. Numerical simulations show that the proposed model obtains almost the same results as the multi-mode model approach under the given conditions. Based on this model, the potential function exhibits an explicit relationship to the post-buckled beam parameters. It shows that the postbuckled beam can be approximated as a snap-through spring-mass system in which the spring stiffness is determined by axial stiffness of beam. As a result, it greatly facilitates the optimization and design of the post-buckled beam generator. Finally, using this simple lumped model, the optimization of a post-buckled beam is performed and its performance is confirmed by comparison with the multi-mode model. 


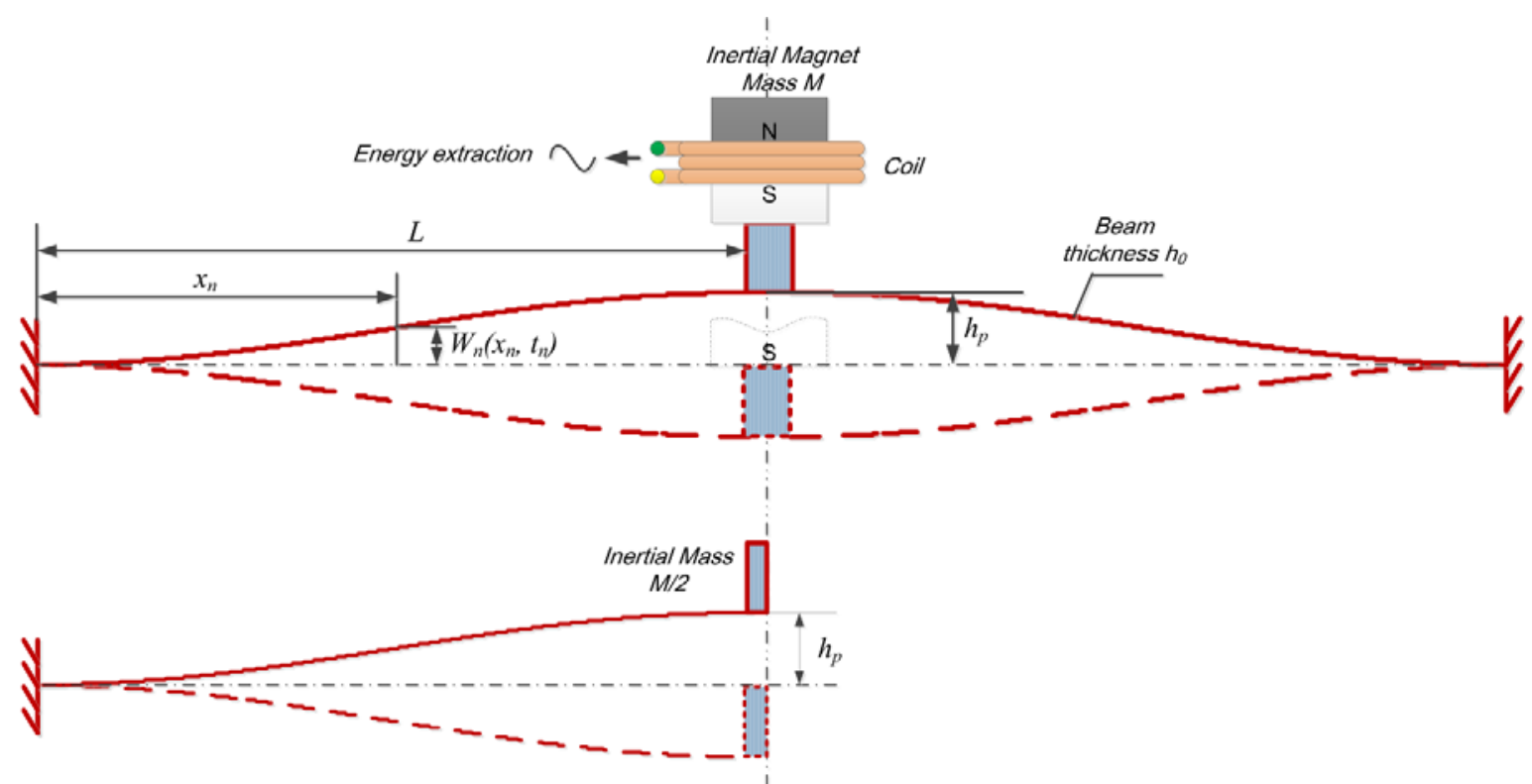

Fig. 1. Schematic of an elementary electromagnetic post-buckled beam harvester

\section{Galerkin and simplified models}

\subsection{Galerkin model}

Considering the symmetry of the structure, only half of the beam is considered. The governing dynamical equation can be written as [42-44]:

$$
m \frac{\partial^{2} W_{n}}{\partial t_{n}{ }^{2}}+E I \frac{\partial^{4} W_{n}}{\partial x_{n}{ }^{4}}+P_{n} \frac{\partial^{2} W_{n}}{\partial x_{n}{ }^{2}}-\frac{1}{2} \frac{E A}{L} \frac{\partial^{2} W_{n}}{\partial x_{n}{ }^{2}} \int_{0}^{L}\left(\frac{\partial W_{n}}{\partial x_{n}}\right)^{2} d x_{n}=-m \frac{\partial^{2} u}{\partial t_{n}{ }^{2}}
$$

with the following boundary conditions

$$
\left.W_{n}\right|_{x_{n}=0}=0 ;\left.\quad \frac{\partial W_{n}}{\partial x_{n}}\right|_{x_{n}=0}=0 ;\left.\quad \frac{\partial W_{n}}{\partial x_{n}}\right|_{x_{n}=L}=0 ;\left.\quad E I \frac{\partial^{3} W_{n}}{\partial x_{n}{ }^{3}}\right|_{x_{n}=L}=\frac{M}{2}\left(\left.\frac{\partial^{2} W_{n}}{\partial t_{n}{ }^{2}}\right|_{x_{n}=L}+\frac{\partial^{2} u}{\partial t_{n}{ }^{2}}\right)-\left.C \frac{\partial W_{n}}{\partial t_{n}}\right|_{x_{n}=L}
$$

in which, $W_{\mathrm{n}}$ is the deflection of the beam, $P_{n}$ is the compression force which is an indication of the beam's initial buckling level, $C=C_{e}+C_{m}$ stands for a combined linear damping associated to the electromechanical convertor $C_{e}$ and the intrinsic mechanical damping $C_{m}$, and $u$ is the excitation movement of the base.

It is pointed out in [45] that eq. (1) derived by Nayfeh et al. [42] is only applied to the case with immovable boundary conditions without accounting for the axial motion which will happen for the unvaried axial force $P_{n}$ with movable ends. However, in this case, $P_{n}$ is not exactly the unvaried axial load, but used to achieve the desired buckled level at the beginning while the boundary condition will be fixed afterwards. The buckling process can be assumed as follows: (1) Firstly, the beam is restricted to have 
only axial deformation without any bending when an axial load is applied; (2) As the axial load increases to $P_{n}$, the beam's two ends are fixed; (3) Finally, the restriction on bending is cancelled and the beam gets buckled with a certain rise $h_{p}$, which is related to the value of $P_{n}$.

Clearly, $P_{n}$ is more specifically the compression force in the case that the post-buckled beam is made flat between two immovable ends while the actual axial force corresponding to the average midline strain can be written as

$$
N=P_{n}-\int_{0}^{L}\left(\frac{\partial W_{n}}{\partial x_{n}}\right)^{2} d x_{n}
$$

which equals the critical Euler buckling load in the post-buckled regime for static analysis as seen in [42, 46-47]. An alternative view of thermal analogy can be helpful to avoid the misunderstanding about the immovable ends and $P_{n}$. The post-buckled beam with the desired rise $h_{p}$ in fig. 1 can be assumed to be induced by applying a temperature change to the clamped-clamped beam. During the whole process, the beam ends are fixed without any movement and $P_{n}$ is still the compression force induced by the temperature change when the beam is flat. Therefore, eq. (1) still holds without axial motion considered. It has to be mentioned that, when the beam ends are movable with a constant axial load, eq. (1) are not applicable any more.

For a more generic discussion, the following dimensionless parameters are introduced:

$$
x=\frac{x_{n}}{L} \quad t=t_{n} / \sqrt{\frac{m L^{4}}{E I}} \quad w=\frac{W_{n}}{r} \quad P=P_{n} \frac{L^{2}}{E I}
$$

Here, $r$ is the gyration radius defined as $r=(I / A)^{1 / 2}$ which equals $h_{0} / 2 \sqrt{3}$ in the case of a rectangular cross section.

Hence, eqs.(1) -(2) are written again as:

$$
\begin{gathered}
\frac{\partial^{2} w}{\partial t^{2}}+\frac{\partial^{4} w}{\partial x^{4}}+P \frac{\partial^{2} w}{\partial x^{2}}-\frac{1}{2} \frac{\partial^{2} w}{\partial x^{2}} \int_{0}^{1}\left(\frac{\partial w}{\partial x}\right)^{2} d x=-\frac{1}{r} \frac{\partial^{2} u}{\partial t^{2}} \\
w_{\mid x=0}=0 ;\left.\quad \frac{\partial w}{\partial x}\right|_{\mid x=0}=0 ; \quad \frac{\partial w}{\partial x_{\mid x=1}}=0 ; \quad{\frac{\partial^{3} w}{\partial x^{3}}}_{\mid x=1}=\alpha\left({\frac{\partial^{2} w}{\partial t^{2}}}_{\mid x=1}+\frac{1}{r} \frac{\partial^{2} u}{\partial t^{2}}\right)-c \frac{\partial w}{\partial t}_{\mid x=1}
\end{gathered}
$$

in which $\alpha=M /(2 m L)$ is the ratio of the inertial mass to total beam mass ratio, and $c=C L / \sqrt{m E I}$.

Noting that if $P$ is greater than the Euler critical force $P_{c}=\pi^{2}$ for boundary conditions associated to this problem, any first-mode buckled configuration can be defined as:

$$
w_{b}=\frac{1}{2} b(1-\cos (\pi x)) \quad \text { where } b=4\left(P-P_{c}\right)^{1 / 2} / \pi
$$

in which $b$ represents the dimensionless buckling level as a function of the force $P$. 
Consequently, the solution of eqs. (5)-(6) can be expressed as:

$$
w(x, t)=w_{b}(x)+v(x, t)
$$

$v(x, t)$ is the time-dependent deflection around the initial dimensionless buckling shape $w_{b}(x)$.

Following the approach of Emam and Nayfeh [42], $v(x, t)$ can be expressed according to the Galerkin mode discretization method:

$$
\begin{aligned}
v(x, t) & =\sum_{i=1}^{N} q_{i}(t) \phi_{i}(x) \\
& =\sum_{i=1}^{N} q_{i}(t)\left(c_{i, 5} \sin \left(s_{i, 1} x\right)+c_{i, 4} \cos \left(s_{i, 1} x\right)+c_{i, 3} \sinh \left(s_{i, 2} x\right)+c_{i, 4} \cosh \left(s_{i, 2} x\right)+c_{i, 5} \cos (\pi x)\right)
\end{aligned}
$$

in which $\phi_{i}$ is the $i^{\text {th }}$ modal shape, $q_{i}(t)$ is the corresponding time-dependent response, $N$ is the considered mode number, $\omega_{i}$ is the $i^{\text {th }}$ natural frequency and

$$
S_{i, 1}=\sqrt{\frac{1}{2}\left(\pi^{2}+\sqrt{\pi^{4}+4 \omega_{i}^{2}}\right)} \quad S_{i, 2}=\sqrt{\frac{1}{2}\left(-\pi^{2}+\sqrt{\pi^{4}+4 \omega_{i}^{2}}\right)}
$$

By doing this, eqs. (5)-(6) become the set of $N$ differential equations:

$$
\ddot{q}_{i}+2 \omega_{i} \xi_{i} \dot{q}_{i}+\omega_{i}^{2} q_{i}-2 b \sum_{j, k=1}^{N} A_{i j k} q_{j} q_{k}-\sum_{j, k, l=1}^{N} B_{i j k l} q_{j} q_{k} q_{l}=F_{i}
$$

in which, $\xi_{i}$ is the damping coefficient of mode $i$ and coefficients $A_{i j k}, B_{i j k l}$ and $F_{i}$ are define as:

$$
\begin{gathered}
A_{i j k}=\frac{1}{8} \pi^{2} \int_{0}^{1} \phi_{i}(x) \cos (\pi x) d x \int_{0}^{1} \frac{\mathrm{d} \phi_{j}}{\mathrm{~d} x} \frac{\mathrm{d} \phi_{k}}{\mathrm{~d} x} \mathrm{~d} x+\frac{1}{4} \pi \int_{0}^{1} \phi_{i}(x) \frac{\mathrm{d}^{2} \phi_{j}}{\mathrm{~d} x^{2}} \mathrm{~d} x \int_{0}^{1} \frac{\mathrm{d} \phi_{k}}{\mathrm{~d} x} \sin (\pi x) \mathrm{d} x \\
B_{i j k l}=\frac{1}{2} \int_{0}^{1} \phi_{i}(x) \frac{\mathrm{d}^{2} \phi_{j}}{\mathrm{~d} x^{2}} \mathrm{~d} x \int_{0}^{1} \frac{\mathrm{d} \phi_{k}}{\mathrm{~d} x} \frac{\mathrm{d} \phi_{l}}{\mathrm{~d} x} \mathrm{~d} x \\
F_{i}=-\frac{1}{r} \frac{\mathrm{d}^{2} u}{\mathrm{~d} t^{2}}\left(\alpha \phi_{i}(x)+\int_{0}^{1} \phi_{i}(x) \mathrm{d} x\right)
\end{gathered}
$$

The multi-mode solution $w(x, t)$ can be then numerically solved using eqs. (11)-(14). For any specific time $t_{0}$, the deflection of the beam at the mid-span point $w\left(x=1, t_{0}\right)$, which is also the displacement of the mass, is then determined. Meanwhile, the potential function $V$ of the post-buckled beam is obtained by substituting the corresponding non-normalized solution $W_{n}\left(x, t_{0}\right)$ into the following potential function [43]:

$$
V=\frac{1}{2} E I \int_{0}^{L}\left(\frac{\partial^{2} W_{n}}{\partial x_{n}{ }^{2}}\right)^{2} d x_{n}-\frac{1}{2} P_{n} \int_{0}^{L}\left(\frac{\partial W_{n}}{\partial x_{n}}\right)^{2} d x_{n}+\frac{E A}{8 L}\left[\int_{0}^{L}\left(\frac{\partial W_{n}}{\partial x_{n}}\right)^{2} d x_{n}\right]^{2}
$$


Hence, the function relationship between $V$ and the displacement of the mass $x_{q}=W_{n}(x=1)$ is established. It is worthy of note that, for the whole post-buckled beam, twice the mechanical energy is to be considered.
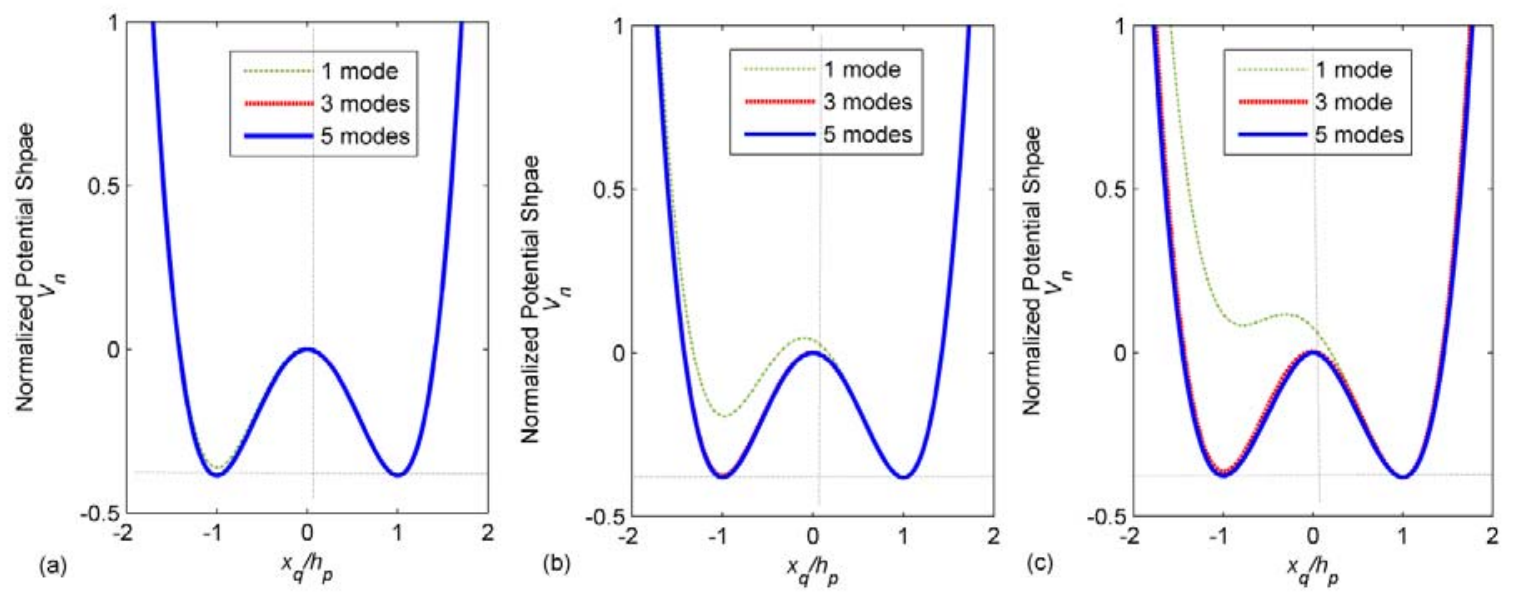

Fig. 2. Post-buckled beam potential shape with different mode numbers considered and various buckling levels: (a) $b=1$; (b) $b=4$; (c) $b=8$.

Fig. 2 shows the evolution of the beam's normalized EPF $V_{n}$ versus the normalized deflection at the mid-span point $x_{q} / h_{p}$ for various buckling configuration $\left(b=h_{p} / r=2 \sqrt{3}\left(h_{p} / h_{0}\right)\right)$ with 1,3 and 5 modes discretization, respectively. For a small buckling level (fig. 2 (a)), the potential shape from the singlemode model is only slightly un-symmetrical with two stable wells. However, as $b$ increases, the symmetry is loss and one of the two stable wells becomes preponderant (fig. 2 (b) and (c)). It is clear that the single-mode model is only acceptable for very small buckling levels. As the buckling level increases, significant discrepancies between the single-mode model, the 3-modes model and the 5-modes model can be seen (fig. 2 (b) and (c)), especially for the lower stable and central unstable positions. In order to have an accurate description of the beam's dynamics over a wide range of buckling levels (for example, $b=0-8$ ), 5 or more modes are to be accounted. However, as we can see, the relationship between potential shape and the beam parameters is not a straight definition using multi-mode model. It is then difficult to use this model directly for the optimization of the EPF of the beam and the optimization of the post-buckled beam generator.

\subsection{Approximation and preliminary comparison}

In order to develop a simple and efficient model, the multi-mode solution is further investigated. The snap-shots of the beam shape at different time and for different initial buckling levels are presented in fig. 3 with the classical Galerkin multi-mode solution. By observing the beam shape of the 5-modes solution and inspired by the studies $[37,38]$ in which the first static buckling mode is used as the single-mode 
solution for the post-buckled beam (actually, the first static buckling mode is not exactly the single-mode solution according to the Galerkin method), an approximate solution for eqs. (5)-(6) is assumed as follows:

$$
w(x, t)=\frac{1}{2} \bar{x}_{q}(1-\cos \pi x)
$$

in which $\overline{x_{q}}=x_{q} / r$ is the dimensionless displacement at the mid-span point $(x=1)$. The corresponding snap-shots of the proposed approximate solution are also plotted in fig. 3 for comparison with the 5modes one. It is obvious that for small and medium buckling levels (fig.3 (a) and (b)), the two solutions are almost the same. As the buckling level increases to a high value $(b=8)$, slight discrepancies are found, especially when the beam is far away from its initial buckling position. Nevertheless, the agreement between two solutions is deemed well enough.
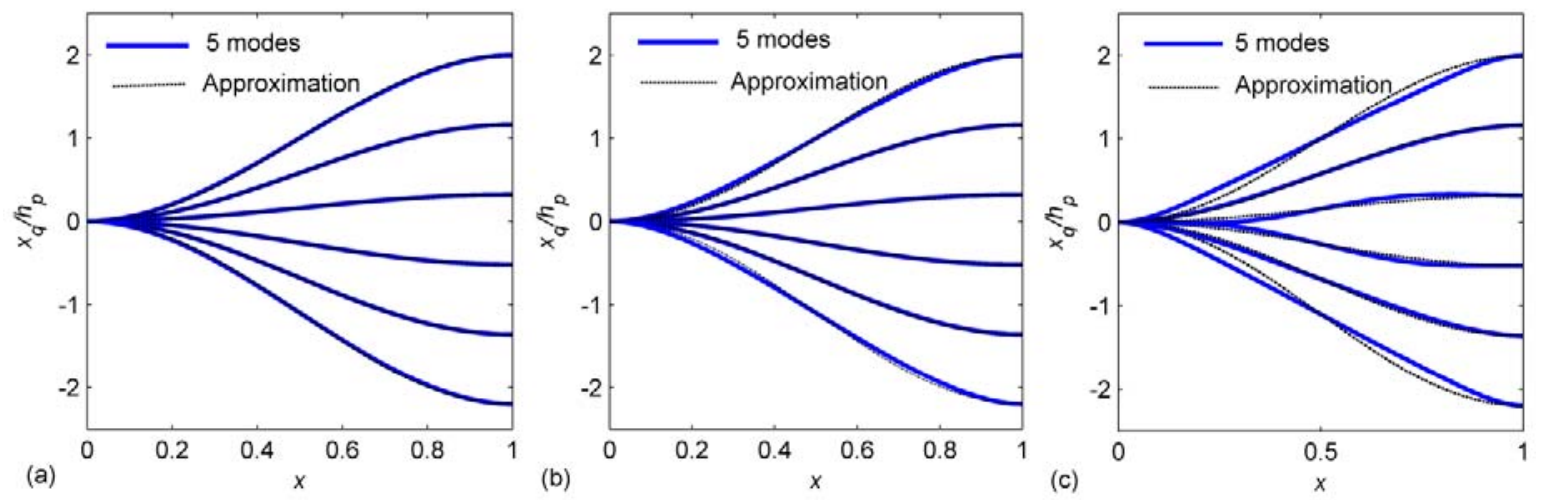

Fig. 3. Snap-shots of the dynamic post-buckled beam for the 5-modes model and the approximate model with various buckling levels: (a) $b=2$; (b) $b=4$; (c) $b=8$.
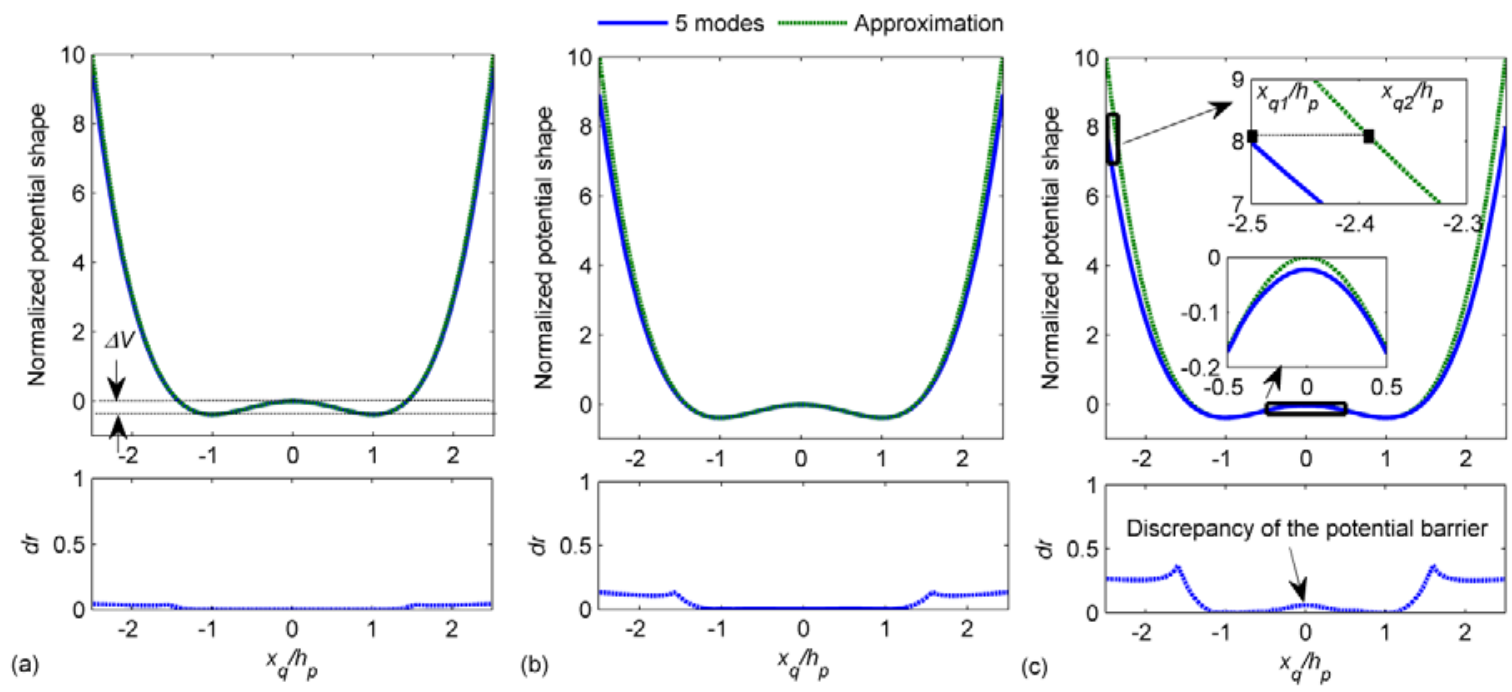

Fig. 4. EPF comparisons for different dimensionless buckling levels between the approximated and the 5modes models: (a) $b=2$; (b) $b=4$; (c) $b=8$. 
Substituting the corresponding non-normalized solution $W_{n}(x, t)=x_{q}(1-\cos (\pi x / L)) / 2$ into the EPF eq. (15) and then multiplying it by two for the total energy, we get:

$$
V_{n}=\frac{E I \pi^{4} x_{q}^{2}}{8 L^{3}}-\frac{P_{n} \pi^{2} x_{q}^{2}}{8 L}+\frac{E A \pi^{4} x_{q}^{4}}{256 L^{3}}
$$

Here, the three terms are related to the bending energy and the axial force and the axial deformation, respectively. With the approximate solution and the 5-modes solution used, the corresponding potential shapes are compared in fig.4. The difference ratio $d r$ is also plotted for each case using the following definition:

$$
d r=\left(V_{2}-V_{1}\right) / \max \left(V_{1}, \Delta V_{1}\right)
$$

where $V_{1}$ is the EPF of the 5 -modes solution, $V_{2}$ is the EPF of the approximate solution and $\Delta V_{1}$ the depth of the potential well for $V_{1}$. The introduction of $\max \left(V_{1}, \Delta V_{1}\right)$ at the denominator allows to avoid the singularity around zero potential.

From fig. 4 (a) and (b), it is confirmed again that the proposed model is consistent for low and medium buckling levels. Even in the strong buckling level (see fig .4 (c) for which $b=8$ ), the difference is small except for the margin zones away from the initial buckling position $\left(x_{q} / h_{p}=1\right)$. Though, if we further inspect the plot in an alternative view of $x_{q}$ 's specific value to reach the same potential value as shown by the zoom window, the difference $\left(x_{q 1}-x_{q 2}\right) / x_{q 1}$ is less than $5 \%$ for the considered range as indicated in fig. 4 (c). Thus, it is inferred that the two models' results are close enough for the dynamic responses.

\subsection{Effectiveness analysis}

Beside the discrepancy between the approximation and the 5-modes model at the margin, it is also found that the difference of the potential barrier's height slightly increases with the buckling level as well, which can be seen in fig. 4 (c). The zoomed picture around $x=0$ shows that the 5 -modes model presents a smaller potential than the approximation one. In comparison with the discrepancy at the margin, the difference of the potential barrier height has a more critical influence since it directly affects the inter-well dynamics and the effectiveness of the approximation solution. Therefore, further analysis is expected to investigate this issue.

As stated at the beginning, eq. (1) - eq. (2) assumes the symmetric situation. Moreover, by carefully checking the approximation solution in eq. (16), it can be drawn that the assumption of symmetric snapthrough has been used and only the first symmetric buckling mode is accounted. However, as the postbuckled rise $b$ increases, the antisymmetric snap-through might coexist with the symmetric one. Furthermore, the high-order symmetric buckling mode might appear as well during the snap-through process. The anti-symmetric snap-through significantly decreases the potential barrier when it plays an important role in the snap-through process as pointed out in [50-52]. 


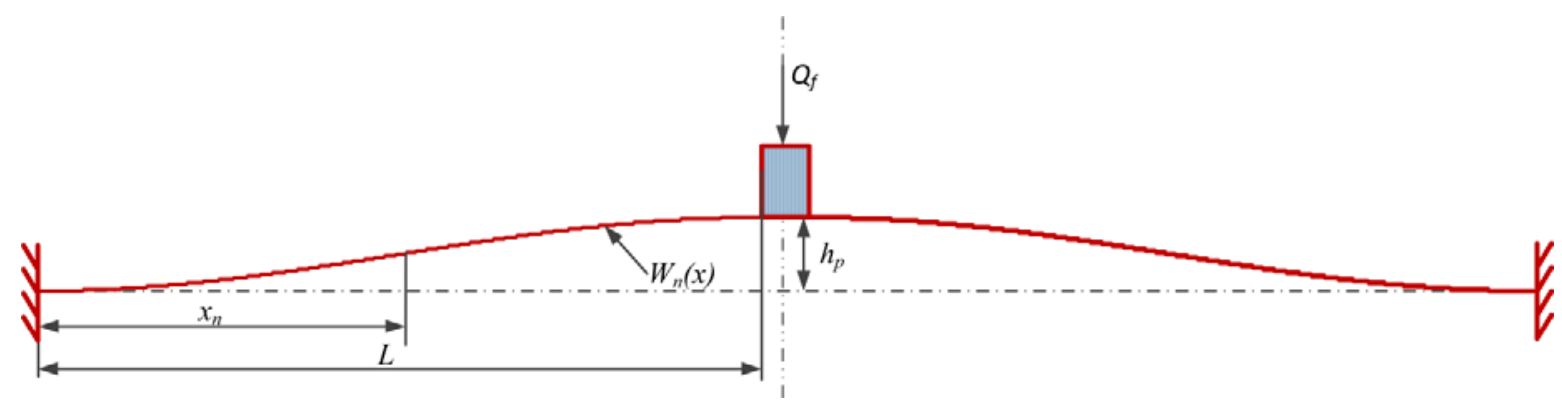

Fig. 5. Post-buckled beam with a distributional load and a concentrated load together.

In order to find out the condition of the antisymmetric snap-through solution, a point force $Q_{f}$ is assumed at the beam's mid-span in fig. 5. Following the approach in [47-48], the nonlinear equilibrium equation can be obtained as:

$$
\frac{\mathrm{d}^{4} w}{\mathrm{~d} x^{4}}+\lambda^{2} \frac{\mathrm{d}^{2} w}{\mathrm{~d} x^{2}}=0
$$

where

$$
\lambda^{2}=P-\frac{1}{2} \int_{0}^{1}\left(\frac{\mathrm{d} w}{\mathrm{~d} x}\right)^{2} d x
$$

stands for the actual axial force $N$ in eq. (3) for the dimensionless case and the boundary conditions are

$$
w_{\mid x=0}=0 ; \quad \frac{\partial w}{\partial x}=0 ; \quad \frac{\partial^{3} w}{\partial x^{3}}=-\frac{\bar{Q}_{f}}{2}
$$

Here, $\bar{Q}_{f}=Q_{f} / r$ is the force normalized by the gyration radius. It is noted that the boundary conditions in eq. (21) is different from the symmetric case in eq. (6) with the slope unrestricted at the beam mid-span. Then we can write the solution as:

$$
w(x)=c_{0}-c_{0} \cos \lambda x+\left(-\frac{\bar{Q}_{f}}{2 \lambda^{2} \cos \lambda x}+c_{0} \lambda \tan \lambda x\right) x+\left(\frac{\bar{Q}_{f}}{2 \lambda^{3} \cos \lambda}-c_{0} \tan \lambda\right) \sin \lambda x
$$

in which $c_{0}$ is the constant related to $\bar{Q}_{f}$. Substituting eq. (22) and the expression of $P$ from eq. (7) into eq. (20) and using the lowest anti-symmetric snap-through condition $\lambda \approx 1.4303 \pi[43,47]$, we have

$$
\bar{Q}_{f} \approx \pm 180.57 c_{0} \pm 2.86 \sqrt{b^{2}-16.74-7.603 c_{0}^{2}}
$$

Considering $c_{0}^{2} \geq 0, b>\sqrt{16.74}=4.09$ is required to have a real solution. It hints that the anti-symmetric snap-through exists only when the normalized buckling level $b$ is larger than 4.09.

Similarly, when the second symmetric buckling mode appears during the snap-through, applying the corresponding buckling condition $\lambda=2 \pi$ [43], we can obtain the solution in this case as

$$
\bar{Q}_{f} \approx \pm 2 \sqrt{\frac{b^{2}-48-16 c_{0}^{2}}{3}} \pi^{3}
$$


The requirement for a possible real solution of the second symmetric buckling mode is therefore $b>6.93$.

Combined the results of eq. (23) and eq. (24), it can be concluded that the accuracy of the snapthrough approximation using the first symmetric buckling mode is ensured for $b<4.09$. By calculating the corresponding compression force $P$ at the buckling level thresholds for the anti-symmetric buckling mode and the second symmetric buckling mode with eq. (7), it is found that the values are identical to the case without the lateral load [43]. It hints that the buckling mode solutions of the beam are only related to the initial mid-span rise or the axial compression force when the beam is made flat.

When the initial buckling level $b$ is higher than the corresponding thresholds, the anti-symmetric buckling or the second symmetric buckling might appear, the potential barrier is lowered obviously during the snap-through of the buckled beam as shown in [50-52]. Fig. 4 (c) presents a direct example of the influence of the second symmetric buckling for a buckling level $b=8$, larger than the threshold 6.93. With the same boundary conditions for symmetric buckling assumption, the 5-modes approach which covers the second symmetric buckling mode shows a relatively lower potential barrier than the approximation approach which only considers the first symmetric buckling as indicated in Fig. 4 (c).

Fig. 6 shows the difference between the potential barriers of the assumed first-order symmetric snapthrough solution $\Delta V_{1}$, the solution with anti-symmetric buckling $\Delta V_{2}$ and the solution with both the first two symmetric buckling modes $\Delta V_{3}$ (with symmetric snap-through only). It is clear that the difference between $\Delta V_{1}$ and $\Delta V_{2}$ increases sharply for $b>4.09$ while the difference between $\Delta V_{1}$ and $\Delta V_{3}$ is observed for $b>6.93$. The approximation solution of eq. (16) has satisfactory accuracy for $b<4.09$. For $b>4.09$, the approximation solution is usable depending on the accuracy threshold. For instance, with an allowed maximum difference of $10 \%$, the buckling level $b=5$ is still acceptable with the anti-symmetric buckling. Moreover, with the anti-symmetric buckling constrained by design, $b<=8$ is usable for the selected threshold of $10 \%$. To be noted, eq. (23) and eq. (24) only give the necessary condition for the antisymmetric and the second-order symmetric buckling respectively. When the lateral load is not meant to excite the anti-symmetric buckling mode or the second-order symmetric buckling load, they will not appear unless internal resonance between two modes happens [42].

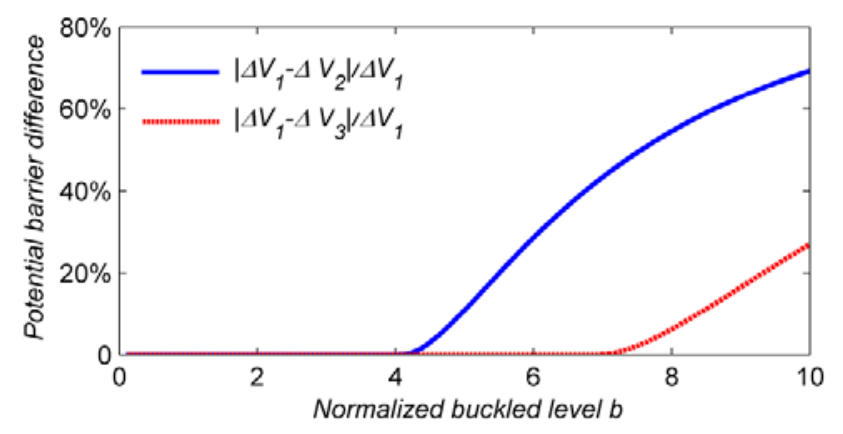

Fig. 6. Difference of the potential barrier height between different snap-though solutions. 


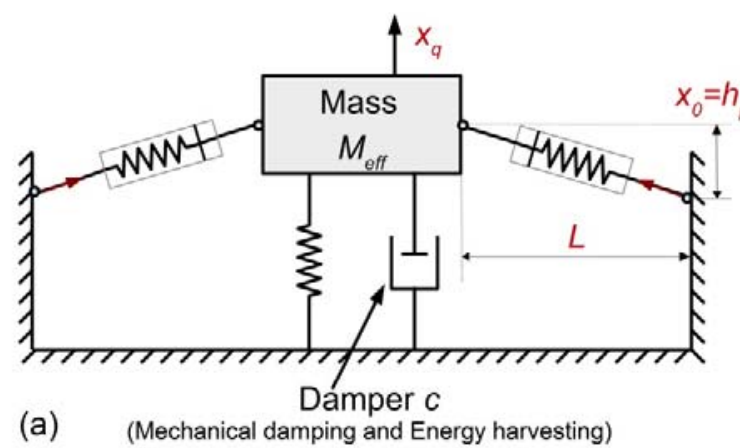

(a)

(Mechanical damping and Energy harvesting)

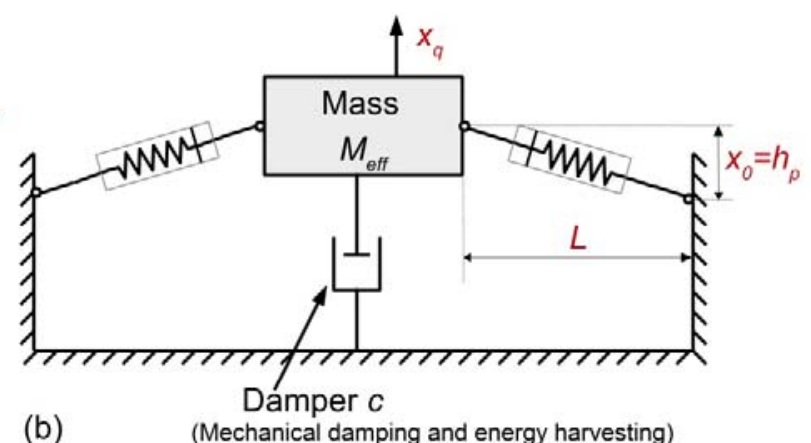

(b)

(Mechanical damping and energy harvesting)

Fig. 7. Simple lumped model for the post-buckled beam: (a) Original form; (b) Reduced form.

\subsection{Simplified lumped model}

When the approximation solution in eq. (16) is applicable for the selected accuracy, a simple lumped model in fig. 7 (a) as an analogy for the post-buckled beam can be established according to the EPF of eq. (17). The vertical spring accounts for the bending stiffness while the horizontal ones represent for the axial stiffness. Considering the common case of $x_{0}=h_{p}<<L$, the EPF is written as:

$$
V_{n s}=\frac{1}{2} K_{r} x_{q}^{2}-\frac{P_{n s} x_{q}^{2}}{L}+\frac{K_{1} x_{q}^{4}}{4 L^{2}}
$$

in which $P_{n s}$ is the compression force of the spring with the mass placed at $x=0$. The model parameters can be identified comparing eq. (25) with eq. (17):

$$
K_{r}=\frac{E I \pi^{4}}{4 L^{3}} \quad K_{1}=\frac{E A \pi^{4}}{64 L} \quad P_{n s}=\frac{\pi^{2}}{8} P_{n} \quad M_{e f f}=M+\frac{3}{4} m L
$$

in which $M_{\text {eff }}$ is the equivalent mass calculated by using the approximate solution eq.(16) for the postbuckled beam. Using the minimum EPF constraint at the initial position $x_{q}=h_{p}$, it holds that:

$$
\left.\frac{d V_{n s}}{d x_{q}}\right|_{x_{q}=h_{p}}=K_{r} h_{p}-2 P_{n s} \frac{h_{p}}{L}+K_{1} \frac{h_{p}^{3}}{L^{2}}=0
$$

Then, we can express the buckling force as:

$$
P_{n}=\frac{4}{\pi^{2}}\left(K_{r} L+K_{1} \frac{h_{p}^{2}}{L}\right)=\frac{E I \pi^{2}}{L^{2}}\left(1+\frac{h_{p}^{2}}{16} \frac{A}{I}\right)=\frac{E I \pi^{2}}{L^{2}}\left(1+\frac{h_{p}^{2}}{16 r^{2}}\right)=\frac{E I \pi^{2}}{L^{2}}\left(1+\frac{b^{2}}{16}\right)
$$

which is exactly the same as the buckling force for the post-buckled beam obtained with the relationship between $P$ and $b$ in eq. (7). It shows that the lump model has good agreement with the model in eqs. (1)(2).

Substitute eq. (28) into eq. (17) or eq. (25) and perform the simplification: 


$$
\begin{aligned}
V_{n}=V_{n s} & =-\frac{E I \pi^{4} b^{2} x_{q}{ }^{2}}{128 L^{3}}+\frac{E A \pi^{4} x_{q}{ }^{4}}{256 L^{3}}=-\frac{E A \pi^{4} x_{q}{ }^{2}}{128 L^{3}} \frac{h_{p}{ }^{2}}{r^{2}} \frac{I}{A}+\frac{E A \pi^{4} x_{q}{ }^{4}}{256 L^{3}} \\
& =-\frac{E A \pi^{4} h_{p}{ }^{2} x_{q}{ }^{2}}{128 L^{3}}+\frac{E A \pi^{4} x_{q}{ }^{3}}{256 L^{3}}=K_{1}\left(-\frac{h_{p}{ }^{2} x_{q}{ }^{2}}{2 L^{2}}+\frac{x_{q}{ }^{4}}{4 L^{2}}\right)
\end{aligned}
$$

Finally, we can write the dynamic equation for the lumped model as:

$$
M_{e f f} \ddot{x}_{q}+C \dot{x}_{q}-K_{1} \frac{h_{p}{ }^{2} x_{q}}{L^{2}}+K_{1} \frac{x_{q}{ }^{3}}{L^{2}}=M_{e f f} \frac{d^{2} u}{d t^{2}}
$$

The available power is:

$$
P_{e}=\frac{1}{2} C_{e} \dot{x}_{q}^{2}
$$

It is worthy of note that from the approximated lumped model, once the initial buckling level $h_{p}$ fixed, the EPF is independent of the beam's bending stiffness $E I$, and only determined by the beam's axial stiffness $E A / L$. In another word, the bending stiffness only influences the buckling force to achieve the desired buckling level. As a result, the model in fig. 7 (a) is identical to the reduced form in fig. 7 (b). The post-buckled beam is then approximated by the simple lumped model composed of the inertial mass and the spring which is only related to the axial stiffness. This feature is especially useful for the design of the post-buckled beam generator with multiple meanings. Firstly, for a given post-buckled rise $h_{p}$ and a constant section area $A$, a section shape design of high moment of inertial $I$ can be chosen so that the normalized buckling level $b=h_{p} r^{-1}=h_{p} A^{1 / 2} I^{-1 / 2}$ can be set to a lower value. In this way, the normalized postbuckled rise $\mathrm{b}$ can be controlled to fall in the effective range of the approximation solution, for instance, $b<=7$ or even $b<=4.09$. The possible solutions of increasing the moment of inertia include increasing the thickness and decreasing the width for the rectangular beam, adopting the I-shape beam, utilizing the double beam structure [49] and so on. The post-buckled rise $h_{p}$ can be then extended to a high value with the simple lumped model still effective. Secondly, more flexibility is expected for the definition of the beam section shape according to specific requirements (i.e. fabrication constraints) with unvarying area $A$. Finally, the proposed lumped model allows an explicit mapping relationship of the EPF and the beam geometrical and material parameters. It greatly reduces the design effort and increases the feasibilities of the design and optimization of a post-buckled beam generator.

Moreover, it can be found that the EPF of the post-buckled beam is mostly driven by the axial deformation while the bending deformation due to the strain difference between the various vertical beam planes can be neglected. This is quite different from the cases of a cantilever or a fixed-fixed beam without pre-stress, in which the bending deformation plays the major role. This is especially relevant when designing bimorph generator with piezoelectric material on each of the beam surfaces. Indeed, for the cantilever beam and the fixed-fixed beam without pre-stress, the piezoelectric material at the top side 
has opposite deformation compared with the one at the bottom side, and the deformation direction changes reversely as the generator crosses the zero position. On the contrary, for a piezoelectric bimorph generator based on post-buckled beam structures, the deformations in the piezoelectric layers at the beam's two sides tend to be the same direction. Moreover, they are symmetrical with respect to the "zero position" so that the induced voltage frequency is two times the excitation frequency in the inter-well motion case as the buckled-spring-mass generator [14, 34].

\section{Numerical validation and generator optimization strategy}

\subsection{Numerical validation}

In order to verify the effectiveness of the proposed simplified lumped model neglecting the bending energy, we perform investigations on two post-buckled beams with different shapes while keeping the sectional area constant. The parameters of these two beams are listed in table 2. It is worthy of note that with the same initial buckling level $h_{p}$ the corresponding dimensionless buckling level is different: $b=2.08$ for beam 1 and $b=3.464$ for beam 2 by varying the moment of inertia with different thickness as mentioned before. The corresponding spring stiffness for the lumped model is calculated as $K_{I}=1.92 \times 10^{7}$ $\mathrm{N} / \mathrm{m}$. Fig. 8 presents the EPF of the two beams using the 5-modes and the simplified lumped models respectively. As expected, despite two different geometrical quadratic moments, similar results are obtained and both of them are close to the equivalent lumped model.

Table 2 Parameters of the two considered post-buckled beams

\begin{tabular}{cccccc}
\hline Parameter & Beam 1 & Beam 2 & Parameter & Beam 1 & Beam 2 \\
\hline$E(\mathrm{MPa})$ & 210000 & 210000 & $M(\mathrm{~g})$ & 31.2 & 31.2 \\
$\rho\left(\mathrm{kg} / \mathrm{m}^{3}\right)$ & 7800 & 7800 & $m(\mathrm{~g} / \mathrm{m})$ & 35.1 & 35.1 \\
$h_{p}(\mathrm{~mm})$ & 0.6 & 0.6 & $A\left(\mathrm{~mm}^{2}\right)$ & 4.5 & 4.5 \\
$L(\mathrm{~mm})$ & 75 & 75 & $I\left(\mathrm{~mm}^{4}\right)$ & 0.375 & 0.135 \\
$W_{t}(\mathrm{~mm})$ & 4.5 & 7.5 & $h_{0}(\mathrm{~mm})$ & 1 & 0.6 \\
\hline
\end{tabular}

Table $31^{\text {st }}$ resonant frequencies of the post-buckled beams with different approaches

\begin{tabular}{ccccc}
\hline Method & Beam 1 & discrepancy & Beam 2 & discrepancy \\
\hline Simplified lumped model & $43.3 \mathrm{~Hz}$ & $5.89 \%$ & $43.3 \mathrm{~Hz}$ & $2.04 \%$ \\
Mode discretization & $43.1 \mathrm{~Hz}$ & $6.52 \%$ & $42.79 \mathrm{~Hz}$ & $3.19 \%$ \\
Finite Element & $46.01 \mathrm{~Hz}$ & - & $44.2 \mathrm{~Hz}$ & - \\
\hline
\end{tabular}


A finite element model simulation has been performed to check for the first-order natural frequencies. From the results in table 3, although the three models show better agreements for beam 2, the error between the discretization method and the simplified lumped model is larger in this case with a higher dimensionless buckling level $b$. The discrepancy between the approximated and the 5-modes solutions one increases with $b$ as pointed out in the previous section. It shows that the approximated model tends to be stiffer than the multi-mode model. In both cases, good agreement is exhibited between the finite element approach, the model-discretization method and the proposed lump model. The results are restricted in a tolerance of $10 \%$ for these three approaches.

Numerical simulations are also performed to investigate the consistence of the proposed model and the multi-mode solution for beam 1 and beam 2. A forward and backward frequency sweep excitation of amplitude $6 \mathrm{~m} / \mathrm{s}^{2}$ from $20 \mathrm{~Hz}$ to $100 \mathrm{~Hz}$ is applied and the displacement responses are presented in fig. 9 . The two beams have close results, except for the discrepancy around the sub-resonant frequency close to the twice the value of the resonant frequency which is underlined in the simplified lumped model. The absence of this sub-resonance in the 5-modes model is thought to be due to the interaction between the different modes as shown in eq. (11).

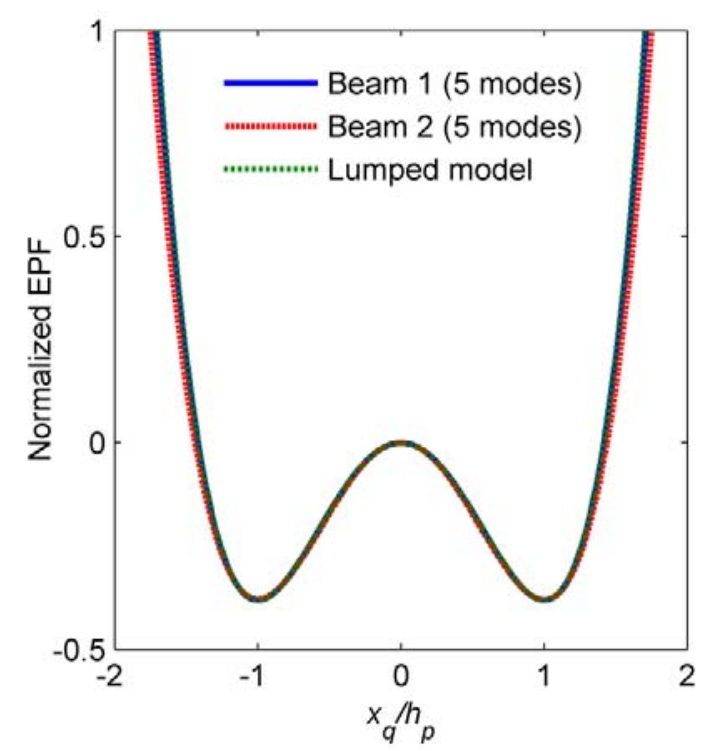

Fig. 8. EPF comparison between the beams with different $E I$ using the 5-modes and the simplified lumped models. 

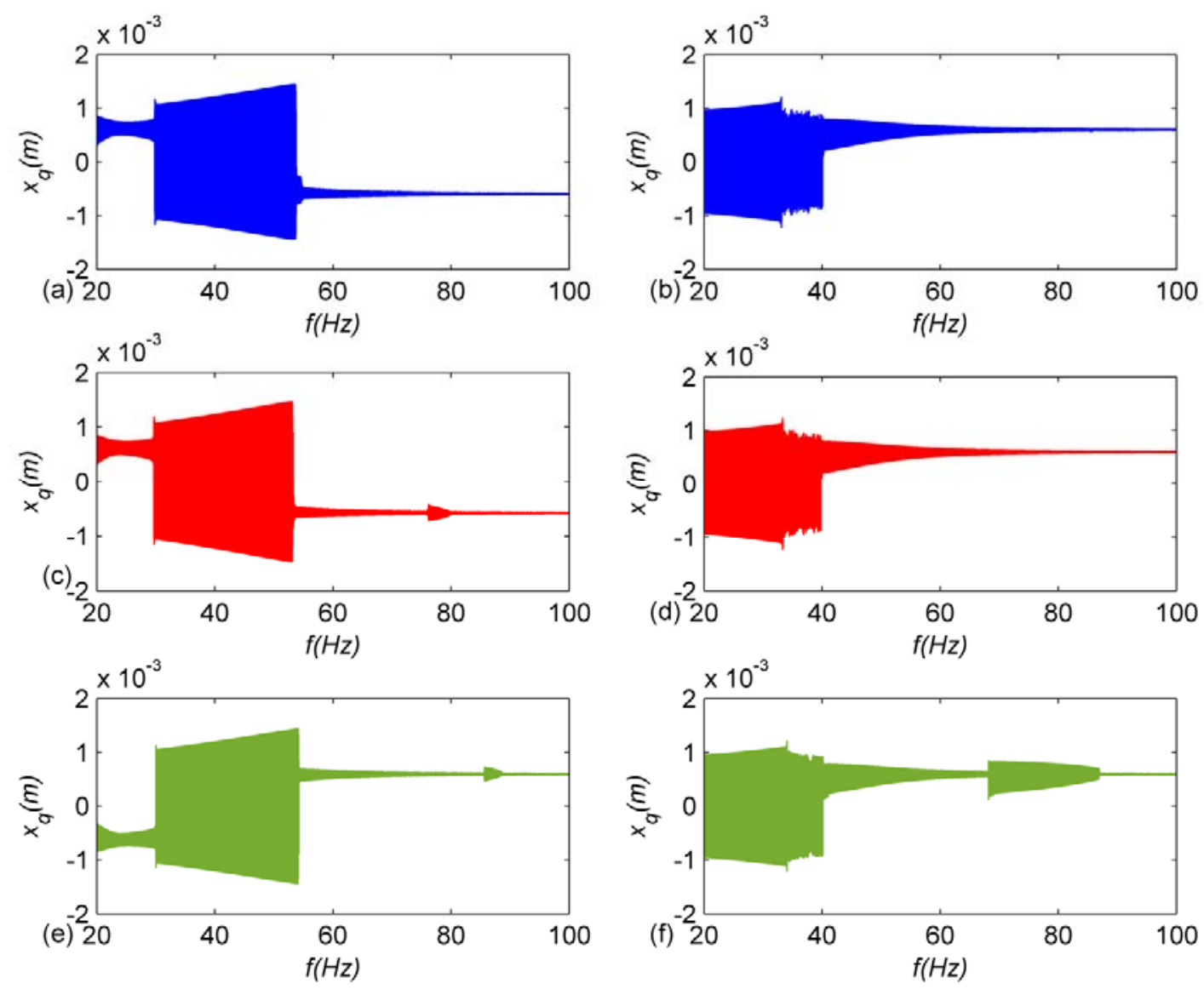

Fig. 9. Displacement responses for beam 1 and 2 using the multi-mode methods and the simplified lumped model. Left: forward sweep, Right: reverse sweep. (a) and (b), beam 1 with 5-modes solution; (c) and (d), beam 2 with 5-modes solution; (e) and (f) simplified lumped model for beam 1 and beam 2.
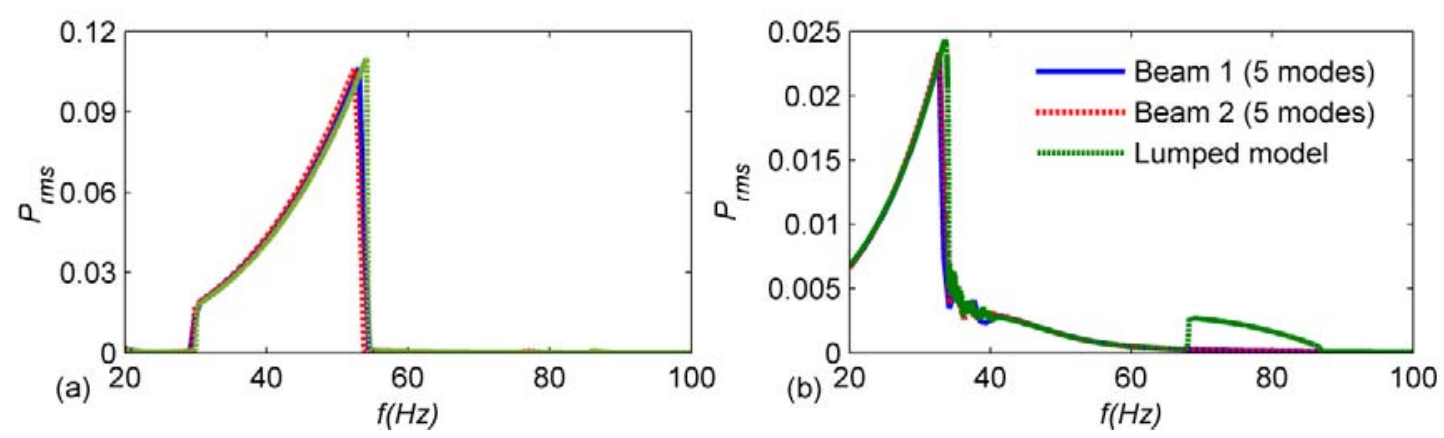

Fig. 10. Nominal power responses for the beam 1 and beam 2 with the multi-mode method and the lump model: (a) forward sweep; (b) reverse sweep.

Considering the sought inter-well dynamics for energy harvesting maximization purpose, the relevance of the simplified lumped model is confirmed whatever the beam's quadratic moment while the interchangeability between the proposed model and the multi-mode model is approved as well. 
As deduced from eq. (31), the generated power is proportional to the square of the velocity and the damping coefficient $C_{e}$. The energy harvesting performances of the two compared generators are evaluated with the RMS value of the nominal power which is defined as follows:

$$
P_{r m s}=\frac{1}{T} \int_{0}^{T} \dot{x}_{q}^{2} d t
$$

in which $T$ is the calculation time period for the RMS value. Fig. 10 clearly presents the amplitude of the power responses. The consistency exhibited by the displacement and the power responses in Fig. 9 and Fig. 10 is a plain validation of the accuracy of the proposed model.

\subsection{Generator optimization strategy}

In order to demonstrate the ability of the simplified lumped model to be used for efficient design, an optimization example is presented. The assumed given excitation signal is the same as before (chirp, $6 \mathrm{~m} / \mathrm{s}^{2}, 20 \mathrm{~Hz}-100 \mathrm{~Hz}$ ), whereas the length of the buckled beam is $2 \times L=150 \mathrm{~mm}$ with an inertial mass of $M=31.2 \mathrm{~g}$, which are usually required to be compliant with some specific application. With the steel material $(E=210 \mathrm{Gpa})$ and the damping coefficient $C$ composed of the mechanical friction $C_{m}$ and the energy harvesting $C_{e}$ assumed, the available optimization parameters of the lump model in eq. (30) are restricted to $K_{l}$ and $h_{p}$, which represent the beam's section area and the initial buckling level respectively.

Prior to the optimization, some mathematics are performed on eq. (30) to aim at a more general discussion. Eq. 30) is written again as:

$$
\ddot{x}_{q}+2 \xi \omega_{0} \dot{x}_{q}-\frac{1}{2} \omega_{0}^{2} x_{q}^{2}+\frac{1}{2} \frac{\omega_{0}^{2}}{h_{p}{ }^{2}} x_{q}^{3}=\frac{d^{2} u}{d t^{2}}
$$

in which

$$
\omega_{0}=\left.\frac{1}{M_{e f f}}\left(\frac{\partial V_{n s}}{\partial x_{q}}\right)\right|_{x_{q}=h_{p}}=\frac{h_{p}}{L} \sqrt{\frac{2 K_{1}}{M_{e f f}}} \quad \zeta=\frac{C}{2 \sqrt{2 K_{1} M_{e f f}}} \frac{L}{h_{p}}
$$

$\xi$ is the damping ratio assumed to be 0.025 on the base of the authors' knowledge and $\omega_{0}$ is the linear resonant frequency for small displacements around the initial buckling position $x_{q}=h_{p}$.

Then, the optimization of the parameters turns to $h_{p}$ and $\omega_{0}$ which is determined by $h_{p}$ and $K_{l}$ together. Besides, considering the time period to be the whole simulation time $T_{a}$, the calculated average nominal power $P_{a v \_r m s}$ can be used to account for the overall performance of the generator. The optimization results with respect to parameters $\omega_{0}$ and $h_{p}$ varying for a selected range are shown in fig. 11.

For a forward sweep in fig. 11 (a), the optimal performance locates at the area of low resonant frequency $\omega_{0}$, and an optimal buckling level is found which is consistent with the studies about the bistable generators $[15,23,25,37]$. As for the reverse sweep in fig. 11 (b), the optimal performance is 
obtained for relatively high $h_{p}$ and $\omega_{0}$ values at the right corner, in which cases the generator motion is stuck in one of the potential well and the response is similar to a softening mono-stable generator [35]. The power peak is due to the softening generator's good performance in the reverse sweep case. Though, the potential harvested power value is much lower than for the forward sweep excitation. A discontinuity is found in both the forward sweep and the reverse sweep cases from the left corner to right corner, which hints the transition from the generators with abundant inter-well motions to the ones with rare inter-well motions (residing in one potential well).

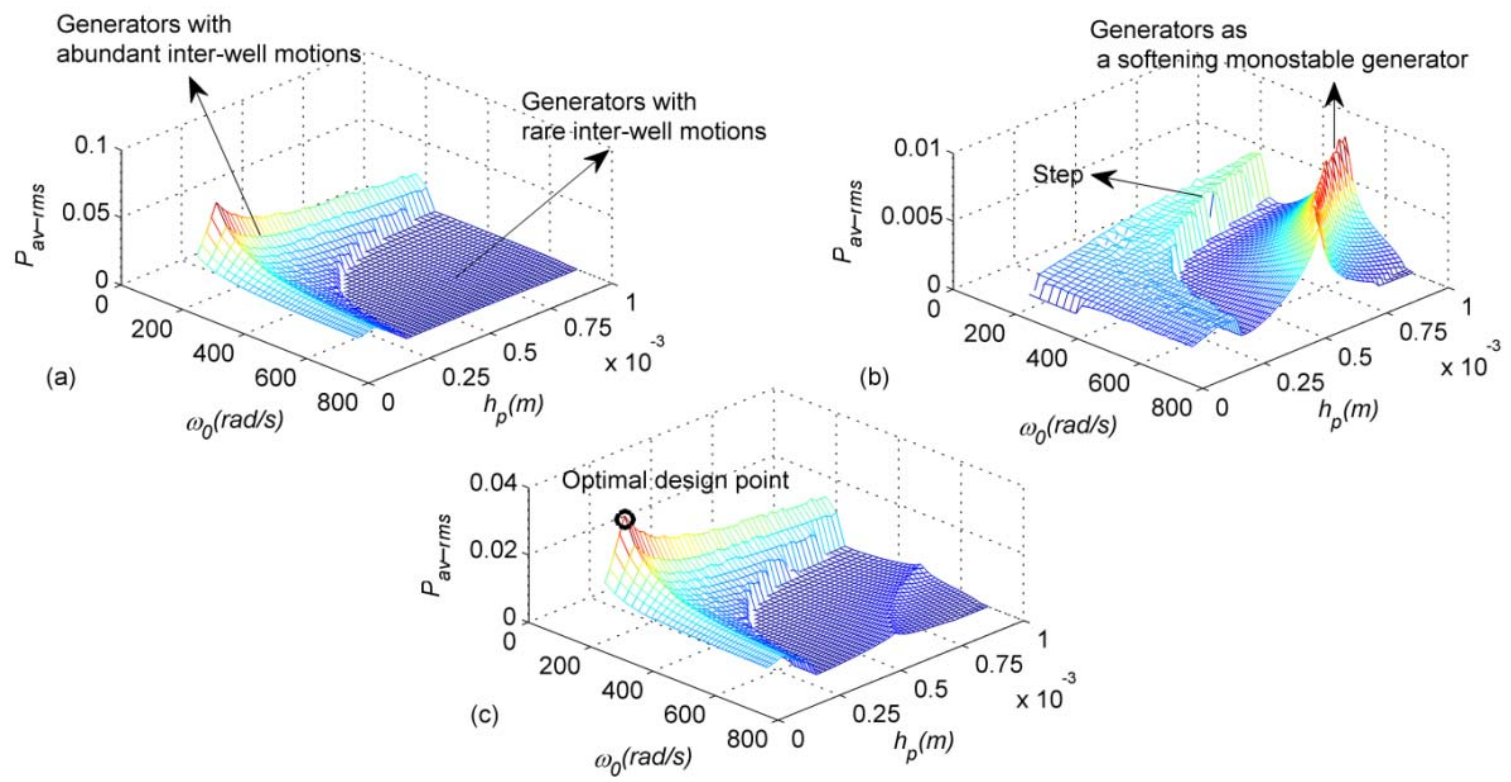

Fig. 11. Average nominal power for different $\omega_{0}$ and $h_{p}$ : (a) forward sweep; (b) reverse sweep; (c) mean value of forward and reverse sweep.

As an overall consideration, the mean nominal power values for the forward and reverse sweep cases are presented in fig. 11 (c). Since a much better performance is obtained in the forward case, the mean results look very similar to fig. 11 (a).

According to the chosen optimal design point $\left(\omega_{0}=158.06 \mathrm{rad} / \mathrm{s}\right.$ and $\left.h_{p}=0.18 \mathrm{~mm}\right)$, we can calculate the corresponding section area $A=W_{t} \times h_{0}=20.44 \mathrm{~mm}^{2}$. As analyzed in section 3, the simplified lumped model provides better approximation when the dimensionless buckling level $b=h_{p} / r=2 \sqrt{3} h_{p} / h_{0}$ is small. Therefore, we could choose the beam parameters as follows: $W_{t}=20 \mathrm{~mm}$ and $h_{0}=1.022 \mathrm{~mm}$ with $b=0.61$ and $M_{\text {eff }}=40.17 \mathrm{~g}$. 

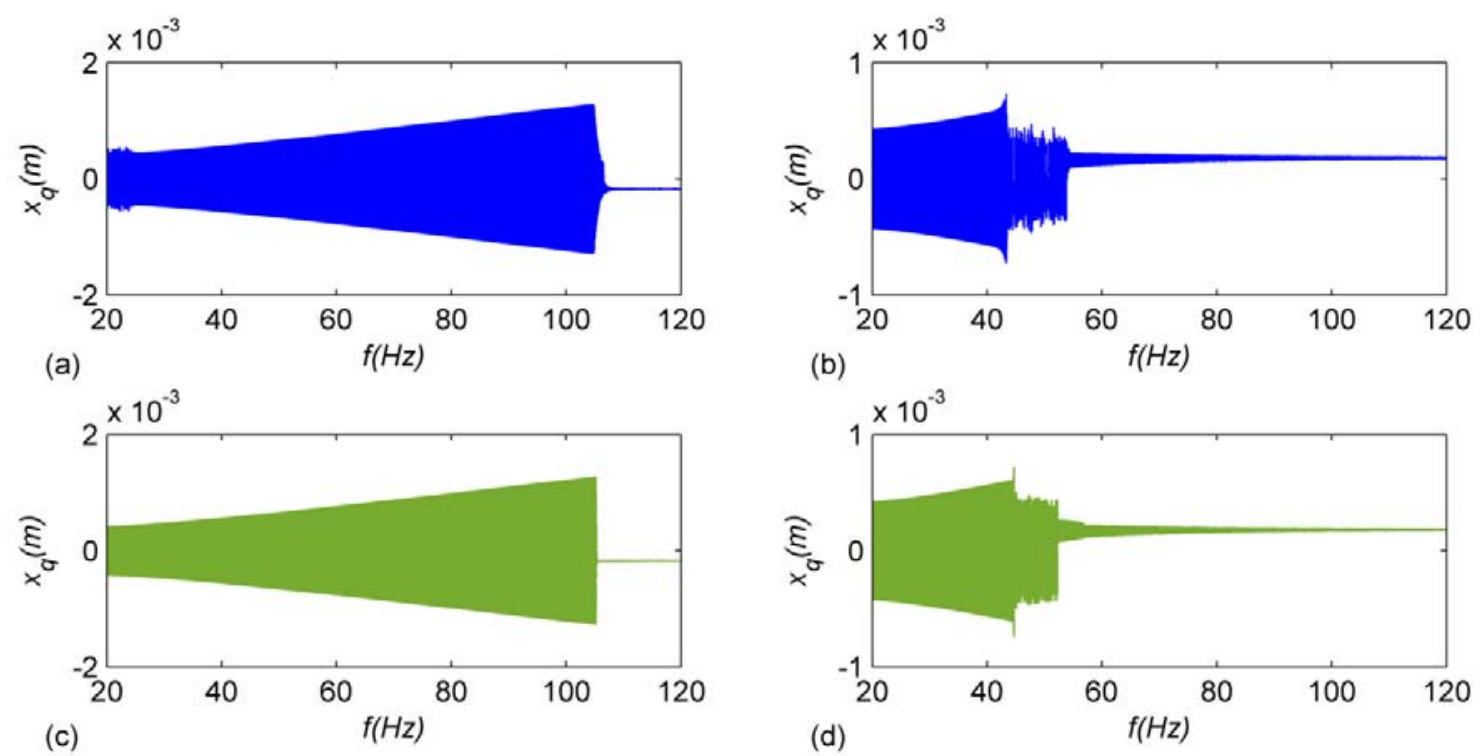

Fig. 12. Displacement responses of the optimal generator design with the model discretization approach and the lump model: (a), (b) 5-modes solution; (c), (d) lump model. Left, forward sweep; Right, reverse sweep.
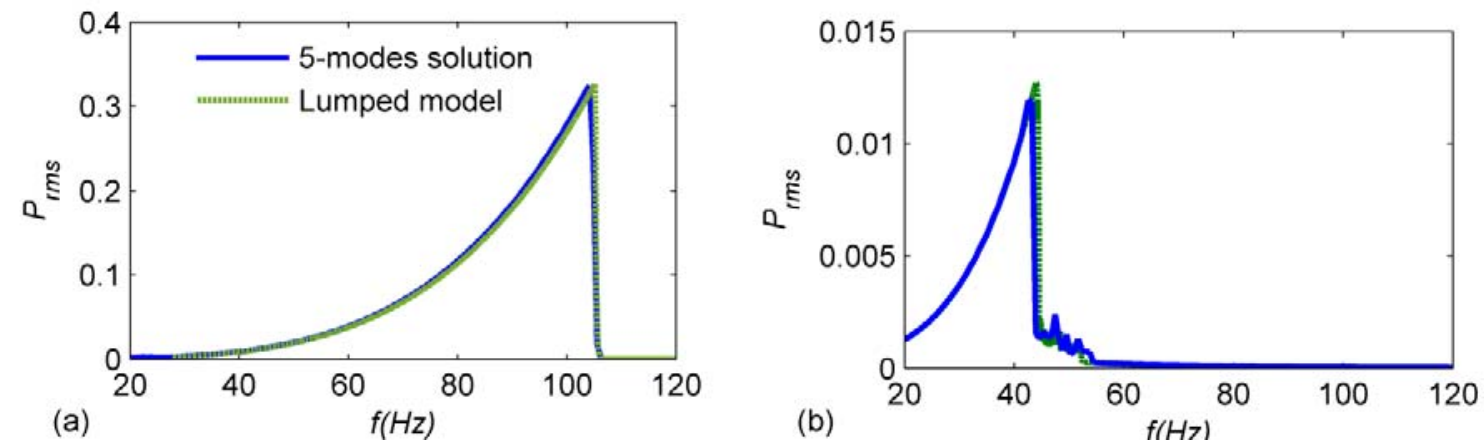

Fig. 13. Nominal power responses of the optimal generator design with the model discretization approach and the lump model: (a) forward sweep; (b) reverse sweep.

Fig. 12 shows again the numerical displacement responses with the Garlerkin discretization method of 5 modes and the simplified lumped model respectively. As we can see, the inter-well motions happen over the whole concerned frequency range for the forward sweep. Even for the reverse sweep, inter-well motions occur for a considerable frequency range. The nominal power responses are also plotted in fig. 13 . The broad bandwidth with the optimal design parameters is clearly observed. Moreover, the agreement between the lump model and the 5-modes solution is once again exhibited. It confirms that the optimization results with the proposed model are reliable enough and equivalent to the outcomes of the multi-mode model with the Galerkin discretization method. The difficulties of using the latter one for the optimization and design of the post-buckled beam generator are therefore solved. The explicit relationship 
of dependence between the beam parameters and the model variables greatly simplifies the optimization process and makes the proposed lump model as a feasible and trustworthy approach.

\section{Conclusion}

As a common realization of the bi-stable generator, the post-buckled beam generator has the advantages of wide bandwidth, compact volume and easy realization. However, the complexity of the regular multimode model obtained with the Galerkin method makes it difficult to be used for optimization and design. The availability of a simple and sufficiently predictive model will ease the post-buckled beam optimization.

Based on the multi-mode model derived for a post-buckled beam generator with an inertial mass mechanical arrangement, a simple lumped model has been proposed. The effectiveness is demonstrated by static as well as dynamic evaluations within a certain normalized buckling range. It shows that the proposed lump model has a good approximation of the multi-mode model, especially in the low dimensionless buckling cases. Moreover, it is observed and underlined that the bending stiffness $(E I)$ has a negligible influence on the beam's energy potential shape and the ensuing dynamics with the buckling level and the section area fixed. It allows the extensive use of the approximation model by selecting a design of high moment of inertial with the section area constant. Moreover, further simplification can be performed and more design space is provided to adapt to the various potential future real applications.

The complexity of using a multi-modes Galerkin approach for the optimization and design of a postbuckled beam generator is therefore skirted. The explicit relationship of dependence between the beam parameters and the model variables greatly simplifies the optimization process. An optimization realistic example is presented to illustrate the applicability of the proposed simplified lumped model.

Obviously, the scope of the model encompasses the post-buckled generator with the electromechanical transducer. Though, further works will aim at designing post-buckled beam generator including piezoelectric layers. This would allow more compact and efficient devices to be obtained.

\section{Acknowledgement}

The authors would like to thank the support from National Natural Science Foundation of China (NSFC) under the grant No.51505395, Sichuan International Cooperation project No.2016HH0028, China Postdoctoral Science Foundation under the grant No. 2016M600747 and Fundamental Research Funds for the Central Universities of China. In addition, the authors would like to give many thanks to the referees for the precious advice on the improvement of this article.

\section{References}

[1] S P Beeby, M J Tudor, N M White, Energy harvesting vibration sources for microsystems applications, Measurement science and Technology 17, R175-R195 (2006). 
[2] L. Tang, Y. Yang, C.K. Soh, Toward broadband vibration-based energy harvesting, Journal of Intelligent Material Systems and structures, 21(18), 1867 (2010).

[3] D. Zhu, M. J. Tudor, S. P. Beeby, Strategies for increasing the operating frequency range of vibration energy harvesters: a review, Measurement Science and Technology, 21(2), 022001 (2010).

[4] J Twiefel, H Westermann, Survey on broadband techniques for vibration energy harvesting, Journal of Intelligent Material Systems and Structures, 24(11), 1291-1302 (2013).

[5] M. F. Daqaq, R. Masana, A. Erturk, D.Dane Quinn, On the Role of Nonlinearities in Vibratory Energy Harvesting: A Critical Review and Discussion, Applied Mechanics Reviews, 66(4), 040801 (2014).

[6] Y. Zhang, C.S. Cai, W. Zhang, Experimental study of a multi-impact energy harvester under low frequency excitations, Smart Materials and Structures, 23(5), 055002 (2014).

[7] M. Pozzi, Impulse excitation of piezoelectric bimorphs for energy harvesting: a dimensionless model, Smart Materials and Structures, 23(4), 045044 (2014).

[8] J.C. Hsu, C.T. Tseng, Y.S. Chen, Analysis and experiment of self-frequency-tuning piezoelectric energy harvesters for rotational motion, Smart Materials and Structures, 23(7), 075013 (2014).

[9] W. Liu, C. Liu, B. Ren, Q. Zhu, G. Hu and W. Yang, Bandwidth increasing mechanism by introducing a curve fixture to the cantilever generator, Applied Physics Letters, 109, 43905 (2016).

[10] L. Tang, Y. Yang, A nonlinear piezoelectric energy harvester with magnetic oscillator, Applied Physics Letters, 101(9), 094102 (2012).

[11] J. Xu and J. Tang, Multi-directional energy harvesting by piezoelectric cantilever-pendulum with internal resonance, Applied Physics Letters, 107, 213902, 2015.

[12] P. Kim and J. Seok, Dynamic and energetic characteristics of a tri-stable magnetopiezoelastic energy harvester, Mechanism and Machine Theory, 94, 41-63, (2015).

[13] E. Halvorsen, G. Litak, Statistics of a noise-driven elastic inverted pendulum, The European Physical Journal: Applied Physics 70(1) 10901 (2015).

[14] W. Liu, F. Formosa, A. Badel, Y. Wu, A. Agbossou, Self-powered nonlinear harvesting circuit with a mechanical switch structure for a bistable generator with stoppers, Sensors and Actuators A: Physical, 216, 106-115 (2014). http://doi.org/10.1016/j.sna.2014.04.040

[15] W. Liu, A. Badel, F. Formosa, Y.P. Wu, N. Bencheikh, A. Agbossou, A wideband integrated piezoelectric bistable generator: Experimental performance evaluation and potential for real environmental vibrations, Journal of Intelligent Material Systems and Structures, 26(7), 872-877, (2015).

[16] M. Rezaeisaray, M. EI. Gowini, D. Sameoto, D. Raboud, W. Moussa, Low frequency piezoelectric energy harvesting at multi vibration mode shapes, Sensors and Actuators A: Physical, 228, 104-111 (2015).

[17] L.J. Gong, Q.S. Pan, W. Li, G. Y. Yan, Y.B. Liu, Z. H. Feng, Harvesting vibration energy using two modal vibrations of a folded piezoelectric device, Applied Physics Letters, 107(3), 033904 (2015).

[18] M. Ferrari, V. Ferrari, M. Guizzetti, D. Marioli, A. Taroni, Piezoelectric multifrequency energy converter for power harvesting in autonomous microsystems, Sensors and Actuators A: Physical, 142:329-335 (2008).

[19] Y. Zhang, K. Liu, L. Tang, Piezoelectric energy harvesting with a nonlinear energy sink, In Proc. of SPIE, 9431, 94310R (2015). http://doi.org/10.1117/12.2084167.

[20] Z. N. Ahmadabadi, S. E. Khadem, Nonlinear vibration control and energy harvesting of a beam using a nonlinear energy sink and a piezoelectric device, Journal of Sound and Vibration, 333(19), 4444-4457 (2014).. http://doi.org/10.1016/j.jsv.2014.04.033.

[21] S.P. Pellegrini, N. Tolou, M. Schenk, J.L. Herder, Bistable vibration energy harvesters: A review, Journal of Intelligent Material Systems and Structures, 24(11), 1303-1312 (2013).

[22] R.L. Harne, K.W. Wang, A review of the recent research on vibration energy harvesting via bistable systems, Smart Materials and Structures, 22(2), 023001, (2013).

[23] F. Cottone, H. Vocca, L. Gammaitoni, Nonlinear Energy Harvesting, Physical Review Letters, 102, 080601 (2009).

[24] A. Erturk, J. Hoffmann, D.J. Inman, A piezomagnetoelastic structure for broadband vibration energy harvesting, Applied Physics Letters, 94(25), 254102 (2009). http://doi.org/10.1063/1.3159815

[25] M. Ferrari, V. Ferrari, M. Guizzetti, B. Andò, S. Baglio, C. Trigona, Improved energy harvesting from wideband vibrations by nonlinear piezoelectric converters, Sensors and Actuators A: Physical, 162(2), 425-431 (2010). http://doi.org/10.1016/j.sna.2010.05.022

[26] S.C. Stanton, C.C. Mcgehee, B.P. Mann, Nonlinear dynamics for broadband energy harvesting: Investigation of a bistable piezoelectric inertial generator, Physica D, 239(10), 640-653 (2010).

[27] A.S. De Paula, D.J. Inman, M.A. Savi, Energy harvesting in a nonlinear piezomagnetoelastic beam subjected to random excitation, Mechanical Systems and Signal Processing, 54-55, 405-416 (2014). 
[28] J. Cao, A. Syta, G. Litak, S. Zhou, D.J. Inman, Y. Chen, Regular and chaotic vibration in a piezoelectric energy harvester with fractional damping, The European Physical Journal Plus, 130(6), 103 (2015).

[29] S. Zhou, J. Cao, D.J. Inman, J. Lin, S. Liu, Z. Wang, Broadband tristable energy harvester: Modeling and experiment verification, Applied Energy, 133, 33-39 (2014). http://doi.org/10.1016/j.apenergy.2014.07.077

[30] B.P. Mann, B. A. Owens, Investigations of a nonlinear energy harvester with a bistable potential well. Journal of Sound and Vibration, 329(9), 1215-1226 (2010). http://doi.org/10.1016/j.jsv.2009.11.034

[31] M. Ferrari, M. Baù, M. Guizzetti, V. Ferrari, A single-magnet nonlinear piezoelectric converter for enhanced energy harvesting from random vibrations, Sensors and Actuators A: Physical, 172(1), 287-292 (2011).

[32] A. Arrieta, P. Hagedorn, A piezoelectric bistable plate for nonlinear broadband energy harvesting, Applied Physics Letters, 97, 104102 (2010). http://doi.org/10.1063/1.3487780

[33] D.N. Betts, H.A. Kim, C.R. Bowen, D.J. Inman, Optimal configurations of bistable piezo-composites for energy harvesting, Applied Physics Letters, 100(11), 114104 (2012). http://doi.org/10.1063/1.3693523

[34] W.Q. Liu, A. Badel, F. Formosa, Y.P. Wu, A. Agbossou, Novel piezoelectric bistable oscillator architecture for wideband vibration energy harvesting, Smart Materials and Structures, 22(3), 035013 (2013).

[35] W. Liu, F. Formosa and A. Badel, Optimization study of a piezoelectric bistable generator with doubled voltage frequency using harmonic balance method, Journal of Intelligent Material Systems and Structures, 28(5), 671686, 2017. http://doi.org/10.1088/0964-1726/22/12/125038

[36] R. Masana, M.F. Daqaq, Relative performance of a vibratory energy harvester in mono- and bi-stable potentials, Journal of Sound and Vibration, 330(24), 6036-6052 (2011).

[37] F. Cottone, L. Gammaitoni, H. Vocca, M. Ferrari, V. Ferrari, Piezoelectric buckled beams for random vibration energy harvesting, Smart Materials and Structures, 21(3), 035021 (2012).

[38] F. Cottone, P. Basset, H. Vocca, L. Gammaitoni, T. Bourouina, Bistable electromagnetic generator based on buckled beams for vibration energy harvesting, Journal of Intelligent Material Systems and Structures, 25(12), 1484-1495 (2013).

[39] A.J. Sneller, P. Cette, B.P. Mann, Experimental investigation of a post-buckled piezoelectric beam with an attached central mass used to harvest energy, Proceedings of the Institution of Mechanical Engineers, Part I: Journal of Systems and Control Engineering, 225(4), 497-509 (2011).

[40] F. Cottone, M. Mattarelli, H. Vocca, L. Gammaitoni, Effect of boundary conditions on piezoelectric buckled beams for vibrational noise harvesting, The European Physical Journal Special Topics, 224(14-15), 2855-2866 (2015). http://doi.org/10.1140/epjst/e2015-02593-5

[41] S.A. Emam, A.H. Nayfeh, On the nonlinear dynamics of a buckled beam subjected to a primary-resonance excitation, Nonlinear Dynamics, 35(1), 1-17 (2004). http://doi.org/10.1023/B:NODY.0000017466.71383.d5

[42] A. Nayfeh, S. Emam, Exact solution and stability of postbuckling configurations of beams, Nonlinear Dynamics, 54(4), 395-408 (2008).

[43] S. A. Emam, A Theoretical and experimental study of nonlinear dynamics of buckled beams (Doctoral dissertation), Virginia Polytechnic Institute and State University (2002).

[44] W. Lacarbonara, A Theoretical and experimental investigation of nonlinear vibrations of buckled beams (Master dissertation), Virginia Polytechnic Institute and State University (1997).

[45] J. N. Reddy and P. Mahaffey, Generalized beam theories accounting for von Kármán nonlinear strains with application to buckling, Journal of Coupled Systems and Multiscale Dynamics, 1(1), 120-134 (2013).

[46] R. H. Plaut, Influence of load position on the stability of shallow arches, Journal of Applied Mathematics and Physics, 30(3), 548-552 (1979).

[47] M. A. Bradford, B. Uy and Y.-L. Pi, In-plane elastic stability of arches under a central concentrated load, Journal of Engineering Mechanics. 128(7), 710-719 (2002).

[48] Y. L. Pi, M. A. Bradford and B. Uy, In-plane stability of arches, International Journal of Solids and Structures. 39(1), 105-125 (2001).

[49] W. Liu, F. Formosa, A. Badel, A. Agbossou and G. Hu, Investigation of a buckled beam generator with elastic clamp boundary, Smart Materials and Structures, 25(11), 115045 (2016).

[50] M. Vangbo, An analytical analysis of a compressed bistable buckled beam, Sensors and Actuators A: Physical, vol. 69 , no. 98 , pp. 212-216 (1998).

[51] R. Wiebe and L. N. Virgin, On the experimental identification of unstable static equilibria, Proceedings of the Royal Society A, 472: 20160172 (2016). http://dx.doi.org/10.1098/rspa.2016.0172

[52] Y. Chandra, R. Wiebe, I. Stanciulescu, L. N. Virgin, S. M. Spottswood and T. G. Eason, Characterizing dynamic transitions associated with snap-through of clamped shallow arches, Journal of Sound and Vibration, vol. 332, no. 22, pp. 5837-5855 (2013). 